\section{Food-motivated performance as a function of weight loss in hypothalamic hyperphagic rats*}

\author{
JOSEPH H. PORTER and JOSEPH D. ALLEN \\ University of Georgia, Athens, Ga. 30601
}

Rats with bilateral ventromedial lesions of the hypothalamus were tested on a VI 1 -min reinforcement schedule at $5 \%, 10 \%, 15 \%$, and $20 \%$ weight loss, first at nonobese then at obese weight levels. During both test phases, rats with lesions of the ventromedial nucleus (VMN) did not differ significantly from the control Ss in response rates, which increased as a linear function of percentage weight loss. However, the VMN Ss' response rates decreased significantly from the nonobese to the obese test phase.

Several studies (Hamilton, 1963; Miller, Bailey, \& Stevenson, 1950; Teitelbaum, 1957) have demonstrated that hypothalamic hyperphagic rats show a lower than normal level of performance in tasks requiring food-directed activity. Falk (1961) reported that dynamic hyperphagic rats had an increased food-motivated response rate on a variable-interval (VI) 1-min reinforcement schedule while maintained at $75 \%-80 \%$ of normal body weight. Marks \& Remley (1972) found that VMN rats reduced to $90 \%$ of control weight levels did not respond less than control rats on a VI 2-min reinforcement schedule. However, the VMN rats reduced to $90 \%$ of static weight responded reliably less than the control rats. Marks and Remley concluded that the performance of VMN rats was related to both the behavioral measure and the body weight of the Ss at the time of testing.

This experiment was designed to answer some of the questions about motivation for food in hypothalamic hyperphagic rats. One important variable appears to be weight of the VMN animal at the time of testing (Falk, 1961; Marks \& Remley, 1972). Another important question involves the relationship between barpressing and percentage weight loss in VMN rats. Collier (1969) has demonstrated that this relationship between response rate and percentage weight loss is an increasing linear function in normal rats. Therefore, Ss were tested at $5 \%$, $10 \%, 15 \%$, and $20 \%$ weight loss in order to provide a more precise comparison of this relationship in VMN rats. The VMN Ss were tested first at nonobese weight levels and then at obese weight levels in order to

*This paper was based upon a thesis submitted by the senior author in partial fulfillment of the requirements for the MS degree in peychology at the University of Geordia. The authors would like to thank Dr. Henry E. Mads for hit axistance, and Sheryl Edmonds for preparation of histological materials. determine the importance of body weight at the time of testing. PROCEDURE

The Ss were 11 Charles River female albino rats, 84 days old at the beginning of the experiment. All Ss were housed individually in stainless steel cages. Two Lehigh Valley Electronics operant conditioning chambers (Model 1417c) with sound-attenuating cubicles were used. The test chamber was illuminated by a $15-\mathrm{V}$ ac houselight, and white noise was present throughout each session. Reinforcers were standard formula 45-mg Noyes pellets.

Following adaptation in the home cages, food (Purina meal) and water intakes and body weights were recorded for 10 days. The mean of the last 5 days was used to establish free-feeding values for each $\mathbf{S}$. Water was available ad lib in the home cages throughout the experiment. The Ss were trained at $90 \%$ of free-feeding weights. After receiving 100

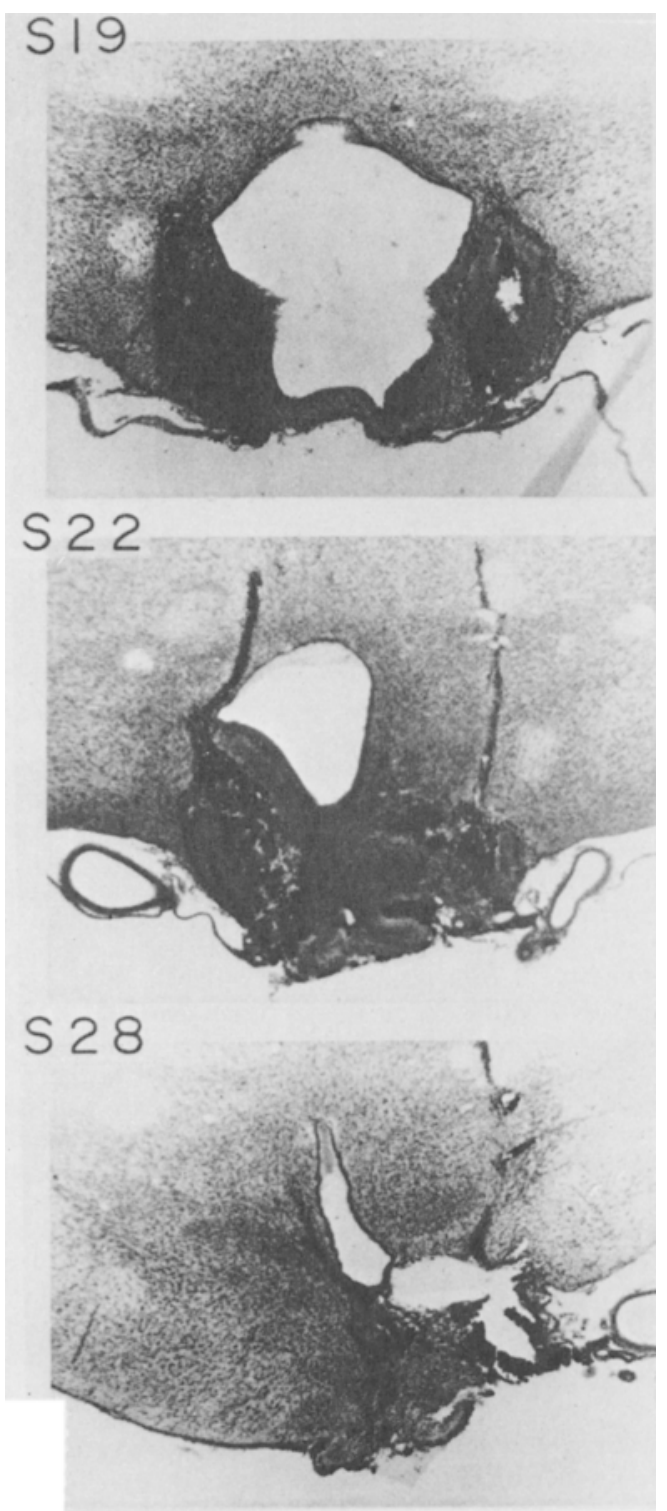

Fig. 1. Representative lesions ranging in size from the largest to the smallest. 


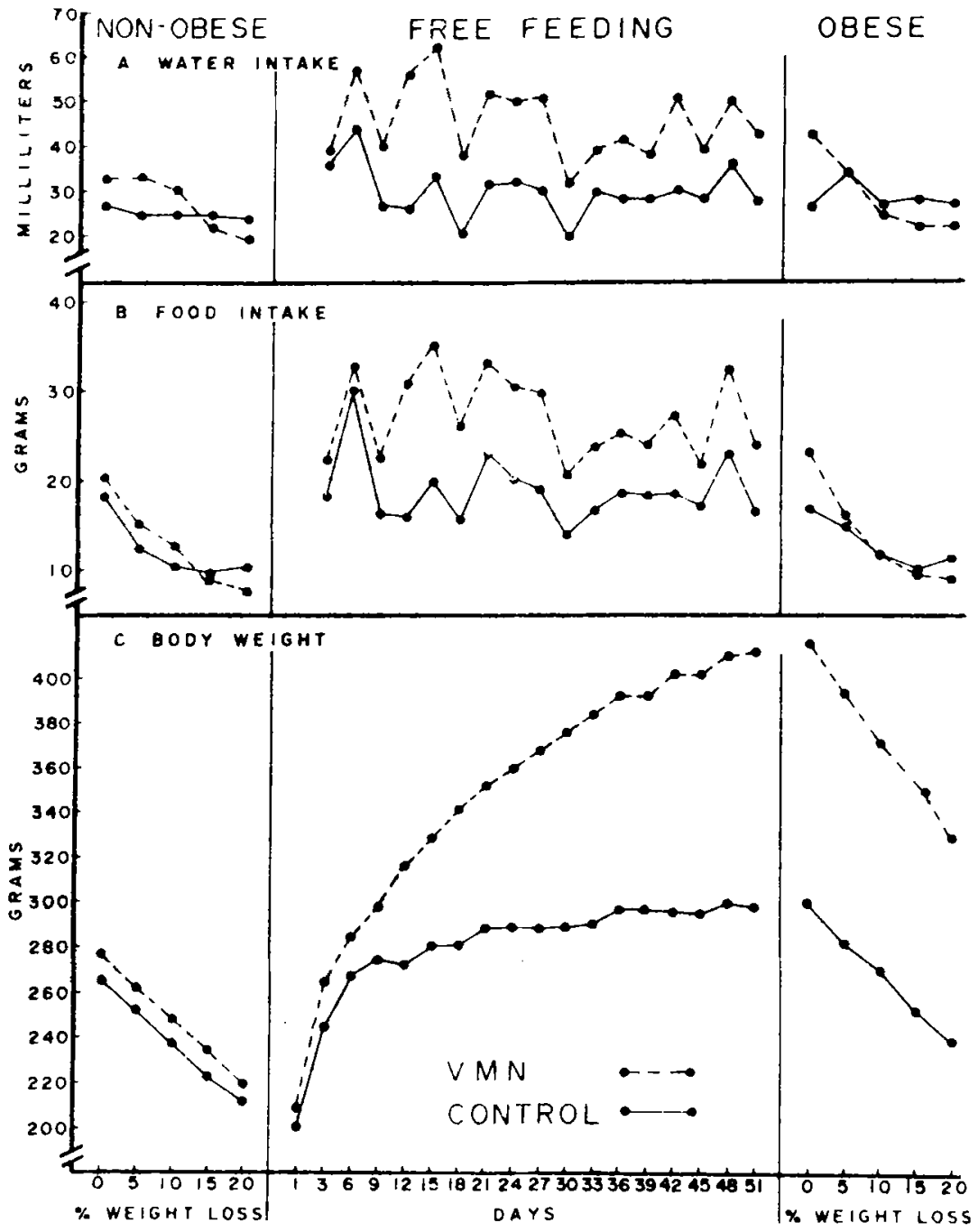

Fig. 2. Body weight, food intake, and water intake for all phases of the experiment.

reinforcers on a continuous reinforcement schedule, each $S$ was given $101 / 2$-h sessions, where responses were reinforced according to a VI 1 -min reinforcement schedule (Fleshler \& Hoffman, 1962). Ss were then allowed to establish new free-feeding weights prior to surgery. The Ss were divided into two groups: six VMN Ss and five control Ss (two were electrode-track controls).

Surgery was performed under sodium pentobarbitol (Nembutal) anesthesia. Bilateral lesions (dc) of the ventromedial nuclei of the hypothalamus (coordinates: $\mathrm{AP}=$ $5.9 \mathrm{~mm}$ from the interaural line, $\mathrm{L}=$ $0.6 \mathrm{~mm}, \mathrm{DV}=0.5 \mathrm{~mm}$ above the base of the skull; the nosebar was set $5 \mathrm{~mm}$ above the interaural line) were produced by passing a current of $1.5 \mathrm{~mA}$ for $15 \mathrm{sec}$ through an elrctrode (stainless steel wire, $.3150 \mathrm{~mm}$ in diam, insulated with Diamel except for $0.5 \mathrm{~mm}$ at the tip).
An anal ground completed the circuit. For the electrode-track controls, the electrode was lowered bilaterally to a position directly above the ventromedial nuclei, but no current was passed. The Ss were returned to their home cages, where water was available, but no food was provided until the next morning.

The nonobese test phase began 2 days after surgery. The Ss were tested for $30-\mathrm{min}$ sessions on the VI $1-\mathrm{min}$ schedule. The testing lasted for 4 days at each of the following consecutive weight-loss levels: $5 \%, 10 \%, 15 \%$, and $20 \%$. Over a 3-day period between each successive weight-loss level, $\mathrm{S}_{\mathrm{s}}$ were deprived of food until the correct weight was obtained. Then they were maintained at that weight level by adjusted daily ration. Following the nonobese test phase, Ss were given free access to Purina meal for 51 days and the VMN Ss were allowed to demonstrate obesity. The obese test phase then began and essentially duplicated the nonobese test phase, except that the-percentage weight loss levels were computed from the new free-feeding weights. Daily food and water intakes and weight measures were recorded throughout the experiment. At the conclusion of the experiment, each operated $S$ was given an overdose of sodium pentobarbitol and perfused with $0.9 \%$ saline, followed by $10 \%$ Formalin. The brains were removed, fixed in Formalin, imbedded in celloidin, and sectioned at $\mathbf{3 0}$ micrometers. Every fifth section was mounted on a slide and stained with thionin.

\section{RESULTS}

All VMN Ss sustained extensive bilateral damage to the ventromedial nucleus of the hypothalamus except for $\mathbf{S} 28$, which had only unilateral damage. Figure 1 shows representative lesions ranging in size from the largest to the smallest. The most accurate and most extensive lesions, which destroyed most of the ventromedial nuclei, were seen in $S 18, S 19$, and $\mathrm{S} 29$. Both S 22 and S 24 had some dorsal ventromedial nuclei intact on one side. Some anterior ventromedial nuclei were remaining in all Ss. The lesions commonly invaded the paraventricular nucleus, the arcuate nucleus, and the most ventral portion of the dorsomedial nucleus. In S 18, the lesion extended posteriorly to include the dorsal and ventral premammilary nuclei. There was no visible damage to the ventromedial nuclei in the electrode-track controls. The three Ss with the most extensive lesions demonstrated the largest weight gains.

Figure 2 shows the body weight, food intake, and water intake data lor all phases of the experiment. The mean preoperative free-feeding weight was $277.0 \mathrm{~g}$ for VMN Ss and $265.3 \mathrm{~g}$ for control Ss. During the free-feeding phase, the VMN Ss gained an average of $139.5 \mathrm{~g}$ over their preoperative weights $(3.1 \mathrm{~g} /$ day $)$. The control Ss gained an average of $34.8 \mathrm{~g}$ over their preoperative weights $(0.8 \mathrm{~g} /$ day $)$. The VMN group had three high weight gainers (mean weight gain equalled $200.7 \mathrm{~g}$ ) and three moderate weight gainers (mean weight gain equalled $78.2 \mathrm{~g}$ ). Analysis of variance revealed no significant differences between the response rates of the two groups. Therefore, the response rates of the high weight gainers and the moderate weight gainers were combined for further analysis.

Analyses of variance were performed on response rates, food intake, and water intake for both the nonobese and the obese test phases. As can be seen in Fig. 2, there was no difference between groups in the 
amount of food and water consumed during either test phase. However, during the free-feeding phase, the VMN Ss consumed daily about $10 \mathrm{~g}$ more food and about $15-20 \mathrm{ml}$ more water on the average than did the control Ss. Food and water intakes decreased reliably (food, $F=110.83$, df $=3 / 27, p<.001$; water, $F=27.26$, df $=3 / 27, p<.001$ ) as percentage weight loss was increased. The Groups by Deprivation interaction was significant for both food intake ( $F=$ $16.34, \mathrm{df}=3 / 27, \mathrm{p}<.001)$ and water intake means $(F=8.54, \mathrm{df}=3 / 27$, $p<.001)$. The slopes of the food and water intake functions were steeper for VMN Ss in both test phases.

Figure 3 shows the response rates for VMN Ss and control Ss during the nonobese and the obese test phases. Response rates for both groups during both test phases increased in a linear and similar manner as percent weight loss increased. During the nonobese test phase, the VMN Ss reponded at higher rates than the control Ss at all four weight-loss percentages; however, the difference between groups did not reach statistical significance. From the nonobese to the obese test phase, the VMN Ss' response rates decreased reliably $(t=2.03, \mathrm{df}=5, \mathrm{p}<.05)$, but the control Ss' response rates did not. For both groups, response rate increased reliably $(\mathrm{F}=17.51$, $\mathrm{df}=$ $3 / 27, p<.001$ ) as percentage weight loss increased, and no difference was found between the slopes of the four functions.

\section{DISCUSSION}

The slopes relating response rate to percentage weight loss did not change for VMN Ss and control Ss between the nonobese and obese test phases. Since the slopes provide an index of relative hunger drive with respect to percentage weight loss, it may be concluded that the relative motivation was similar across groups. Absolute changes in response rate at comparable levels of percentage weight loss may also be used as an index of motivation. Statistical analysis found no significant between-group differences on this measure. This finding is contrary to reports by Miller et al $(1950)$ and Teitelbaum (1957), who found a decrement in VMN animals' response rates. It is in partial agreement with Marks \& Remley's (1972) findings. They found no significant difference in response rates between the VMN rats reduced to $90 \%$ of control weight levels and the control rats. However, they did find a decrement in the obese VMN rats' response rates from the controls' response rates.

Falk (1961) has reported an increase in food-motivated response rate for VMN rats. During the nonobese test phase, the VMN's mean

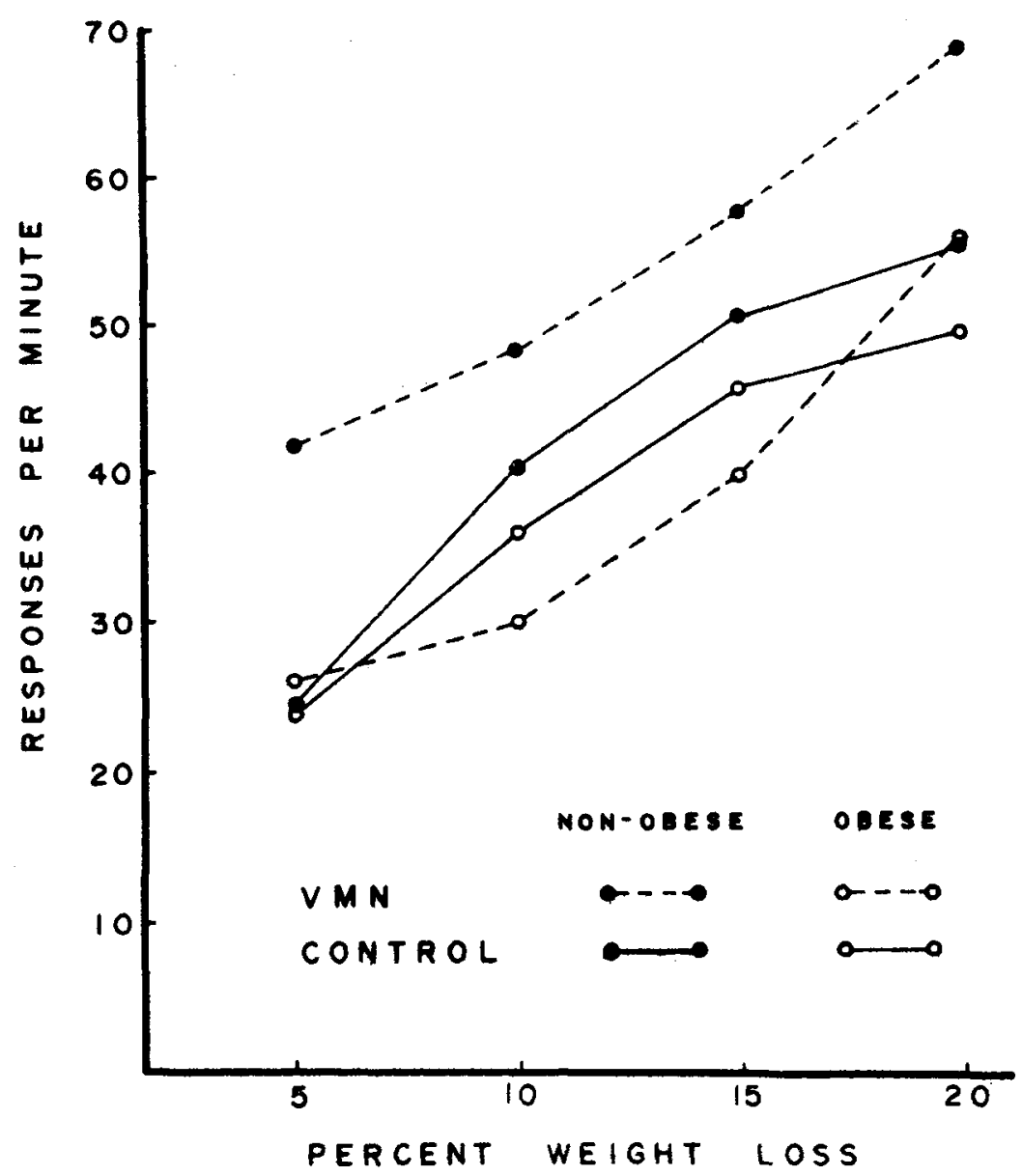

Fig. 3. Mean response rates as a function of percentage weight loss for VMN and control Ss during the nonobese and the obese test phases.

response rate was higher than the control's at all four weight-loss levels (see Fig. 3). However, these differences were not statistically significant. A more powerful within-group design, such as used by Falk (1961), might reveal that the increase in response rates for VMN rats found in this study is reliable.

A within-group comparison made on VMN Ss revealed a significant decrease in absolute response rates from the nonobese to the obese test phase. This drop in response rates may be attributed to either a decrease in absolute motivation for food when in an obese state or some incapacitating effect of excessive weight. Marks \& Remley (1972) suggested that an effort variable due to excessive weight might account for the differences between nonobese and obese VMN rats, but concluded that weight alone would not account for the differences. Since this study found that the obese VMN Ss' response rates were not different from those of the nonobese controls, a strict account in terms of a weight variable alone was not plausible.
The thermostatic theory of hunger (Brobeck, 1948) states that eating is regulated by body temperature, with eating being suppressed by high temperatures and enhanced by low temperatures. It has been suggested that there is a positive relationship between an animal's body weight and hyperthermia, induced by exercise (Han \& Brobeck, 1961), and that a test situation that required an obese animal to work for food (e.g., barpressing) might confound food intake and temperature regulation (Hamilton, 1963). According to this theory, a nonobese VMN rat should be able to maintain a lower body temperature than an obese VMN rat while barpressing and should therefore be able to sustain a higher response rate. The results of this study support this proposal insofar as there was a significant deerease in the VMN's response rates from the nonobese to the obese test phase. However, there also should have been a significant decrease in the obese VMN's response rates from those of the control $\mathrm{Ss}$ during the obese test phase. This finding was not obtained. If the VMN 
Ss had been allowed to reach their static weights, a clear response-rate deficit may have been obtained.

The glucostatic (Mayer, 1953) and the lipostatic (Kennedy, 1953) theories of hunger propose that eating is regulated by chemoreceptors in the central nervous system that are sensitive to circulating metabolites in the blood. If the glucostat or lipostat is altered (e.g., by a VMN lesion), the animal should regulate its weight at a new base level, which would be elevated in the case of a VMN lesion. If excessive weight gain was then prevented following the lesion, both theories would predict that the VMN animal should demonstrate a higher motivation for food than should a normal animal since the VMN animal's body weight deviates more from its new base weight level. Also, if the VMN animal were permitted to reach its new obese-weight level, food-motivated behavior should be similar to that of a normal animal. As would be predicted by these two theories, VMN rats did maintain a higher response rate than did the control rats during the nonobese test phase, but the difference was not statistically significant. During the obese test phase, the VMN and control rats' response rates were not significantly different, as predicted from the theories. The finding that relative motivation (the slopes of the response rate/percentage weight loss functions) varied independently of such factors as the presence or absence of the VMN lesion and the obesity of the animals represents an invariency that should be accounted for in any viable theory of hunger regulation.

\section{REFERENCES}

BROBECK, J. R. Food intake as a mechanism of temperature regulation. Yale Journal of Biology \& Medicine, $1948,20,545$.

COLLIER, G. Body weight loss as a measure of motivation of hunger and thirst. Annals of the New York Academy of Sciences, 1969, 157, 594-609.

FALK, J. L. Comments on Dr. Teitelbaum's paper. In M. R. Jones (Ed.), Nebraska symposium on motivation. Lincoln: University of Nebraska Press, 1961.

FLESHLER, M., \& HOFFMAN, H. S, A progression for generating variable interval schedules. Journal of the Experimental Analysis of Behavior, 1962, 5, 529-530.

HAMILTON, C. L. Interactions of food intake and temperature regulation in the rat. Journal of Comparative \& Physiological Psychology, 1963, 56 , 476-488.

HAN, P. W., \& BROBECK, J. $\mathbf{R}$. Temperature regulation of rats with hypothalamic hyperphagia. American Journal of Physiology, 1961, 200, 703-706.

KENNEDY, G. C. The role of depot fat in the hypothalamic control of food intake in the rat. Proceedings of the Royal Society, B, 1953, 140, 578-592.

MARKS, H. E., \& REMLEY, N. R. The effects of type of lesion and percentage body weight loss on measures of motivated behavior in rats with hypothalamic lesions. Behavioral Biology, 1972, 7, 95-111.

MAYER, J. Glucostatic mechanism of regulation of food intake. New England Medical Journal, 1953, 249, 13-16.

MILLER, N. E., BAILEY, C. J., \& STEVENSON, J. A. Decreased "hunger" but increased food intake resulting from hypothalmic lesions. Science, 1950, 112, $256 \cdot 259$.

TEITELBAUM, P. Random and food-directed activity in hyperphagic and normal rats. Journal of Comparative \& Physiological Psychology, 1957, 50 . $486-490$. 